\title{
Varietas Indiana : le cas de la Miscelánea Antártica de Miguel Cabello Valboa
}

Varietas Indiana: el caso de la Miscelanea Antartica de Miguel Cabello Valboa

Varietas Indiana: the case of Miscelanea Antartica de Miguel Cabello Valboa

\author{
Sonia V. Rose
}

\section{CpenEdition}

Journals

Édition électronique

URL : http://journals.openedition.org/bifea/6886

DOI : $10.4000 /$ bifea.6886

ISSN : 2076-5827

Éditeur

Institut Français d'Études Andines

Édition imprimée

Date de publication : 1 décembre 2001

Pagination : 413-425

ISSN : 0303-7495

\section{Référence électronique}

Sonia V. Rose, «Varietas Indiana : le cas de la Miscelánea Antártica de Miguel Cabello Valboa », Bulletin de l'Institut français d'études andines [En ligne], 30 (3) | 2001, mis en ligne le 08 décembre 2001, consulté le 10 décembre 2020. URL : http://journals.openedition.org/bifea/6886 ; DOI : https://doi.org/ $10.4000 /$ bifea.6886

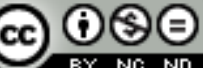

Les contenus du Bulletin de l'Institut français d'études andines sont mis à disposition selon les termes de la licence Creative Commons Attribution - Pas d'Utilisation Commerciale - Pas de Modification 4.0 International. 


\title{
VARIETAS INDIANA : LE CAS DE LA MISCELÁNEA ANTÁRTICA DE MIGUEL CABELLO VALBOA
}

Sonia V. ROSE*

\begin{abstract}
Résumé
La notion de varietas est reprise par la culture de l'Humanisme et érigée en principe générateur de formes de pensée et d'écriture. Compte tenu de ceci, il y a lieu de se demander quel fut son rôle dans l'appréhension intellectuelle du Nouveau Monde et dans les divers discours sur celui-ci, aussi bien ceux qui furent élaborés depuis l'Europe que ceux qui le furent depuis les Indes Occidentales. Il s'agit ici de réfléchir sur un cas particulier, celui de la fonction de la varietas dans la Miscelánea Antártica de Miguel Cabello Valboa, pour essayer de démontrer comment l'auteur se sert doublement de ce principe pour insérer le lecteur, aussi bien local que peninsulaire, au sein de la matière américaine et, en même temps, assimiler celle-ci au fonds commun du savoir, rattachant l'histoire indigène à l'Histoire universelle.
\end{abstract}

Mots clés : Cabello Valboa, varietas, humanisme, histoire de l'Amérique espagnole coloniale.

\section{VARIETAS INDIANA: EL CASO DE LA MISCELÁNEA ANTÁRTICA DE MIGUEL CABELLO VALBOA}

\section{Resumen}

La noción de varietas se erige como principio generador de formas de pensamiento y de escritura y, en un sentido general, como eje de la cultura del Humanismo. Teniendo en cuenta lo anterior, cabe preguntarse sobre la función que ésta cumplió en la aprensión intelectual de los territorios ultamarinos dentro del imaginario europeo y en la formación de un imaginario local. En el presente artículo hemos examinado esta cuestión en un caso particular, el de la Miscelánea Antártica de Miguel Cabello Valboa, intentando mostrar cómo el autor se sirve doblemente del principio de la varietas para insertar al lector, tanto local como peninsular, en el ámbito de la materia americana y de la historia indígena, pero sobre todo para asimilarlas al fondo común del saber y engarzarlas, en un plano de igualdad, dentro de la historia universal.

Palabras claves: Cabello Valboa, varietas, humanismo, historia americana colonial.

\footnotetext{
* Université de Paris-Sorbonne. Pour communication, écrire : 13, rue de Loing 75014 Paris. E-mail : svr75014@yahoo.com
} 


\title{
VARIETAS INDIANA: THE CASE OF MISCELÁNEA ANTÁRTICA DE MIGUEL CABELLO VALBOA
}

\begin{abstract}
The age of Humanism revived the notion of varietas and established it as a generating force of thought and literature. It is therefore necessary to examine the role of varietas in the intellectual conception of the New World, both in Europe and the recently discovered territories. This article studies a specfic case, that of the Miscelánea Antártica of Miguel Cabello Valboa. It sets out to show how this author makes a double use of the principle of varietas in order to place the reader, both local as well as Spanish, within the context of American themes and of native history. However, his principal goal is that of incorporating American indigenous history into the mainstream of Universal History.
\end{abstract}

Key words: Cabello Valboa, varietas, Humanism, Latin American Colonial History.

Pour justifier la composition de son Jardín de flores curiosas (Salamanca, 1570), Antonio de Torquemada signale dans sa dédicace à don Diego Sarmiento de Sotomayor, évêque d'Astorga, la nécessité de rendre compte de la variété des choses de la nature. Tâche tout à fait nécessaire, laisse entrevoir l'auteur, surtout à une époque où l'univers semble être en perpétuelle expansion, apportant chaque jour des nouveautés à sa porte (1). Son oeuvre, comme celle de Pedro Mexía et de quelques autres qui le précédèrent, prétend donc rendre compte de la varietas, à un degré bien supérieur à ce que les Anciens ont pu imaginer et — nous touchons ici le point le plus important chez Torquemada fournit la preuve de l'universalité de Dieu.

Les découvertes et les conquêtes révélèrent et apportèrent à l'horizon européen des preuves constantes de la variété de l'homme et de la nature, et la nécessité de la représenter (2). Cela exigea une adaptation de l'univers intellectuel européen et engendra des processus complexes d'assimilation de l'inconnu à l'intérieur de modèles existants, forcément rénovés et transformés par les nouveaux apports. C'est à ce moment-là que la notion de varietas est reprise et érigée en principe générateur de formes de pensée et d'écriture et, plus généralement, comme l'axe de la culture de l'Humanisme (3). Compte tenu de ce qui vient d'être dit, il y a lieu de se demander quel

(1) "La nature est si puissante et présente des choses si variées, le monde est si grand que chaque jour un grand nombre de nouveautés parviennent à notre connaissance, parmi lesquelles V.S.R. [...] se réjouira d'en voir ici plusieurs d'entre elles réunies, avec d'autres matières curieuses et singulières" (Torquemada, 1982 : 96). Voir Rallo Gruss (1984); sur Torquemada, p. 172 et suiv.

(2) Il n'y a pas lieu de poser ici, mais seulement de signaler, la question polémique de la réception et du plus ou moins grand impact des Indes Occidentales sur l'humanisme espagnol. Sur la question des miscellanées, voir Rodríguez Cacho (1991).

(3) Voir les travaux présentés à la Journée d'étude de l'École des chartes de 2000, consacrée à la varietas. (Varietas/ De varietate rerum à la Renaissance. Études réunies par Dominique de Courcelles, 2001). En constatant la permanence et la pertinence de la varietas en philologie, littérature, philosophie de la nature, savoir théologique et expérience mystique, architecture et musique européennes, ces études montrent le rôle central de cette dernière dans les discours (re)fondateurs des savoirs. 
fut le rôle de la notion de varietas dans l'appréhension intellectuelle des territoires d'outre-mer, dans quel sens elle aida ou fit obstacle à leur incorporation à l'imaginaire européen. Il conviendrait également de se demander quelle fonction elle remplit dans les divers discours sur les Indes Occidentales, aussi bien ceux qui furent élaborés depuis l'Europe (ceux d' humanistes comme López de Gómara ou Calvete de Estrella) que ceux qui le furent depuis les Indes ou par ceux qui avaient une grande expérience de ces régions (tel est le cas de Gonzalo Fernández de Oviedo). Le but de l'étude que nous présentons ici est de réfléchir sur la fonction de la varietas dans un cas en particulier, celui de la miscellanée de Miguel Cabello Valboa (1951)(4).

Parmi le corpus relativement limité des oeuvres composées dans la vice-royauté du Pérou à la fin du XVIe siècle, trois au moins sont à caractère miscellanique : àl'oeuvre de Cabello Valboa déjà mentionnée s'ajoutent la Miscelánea austral de Diego Dávalos y Figueroa (1602-1603) (5) et la traduction faite par Enrique Garcès de De Regno de Francisco Patrizzi (6).

La Miscelánea antártica première oeuvre écrite en espagnol intitulée miscellanée, fut rédigée entre 1576 et le 9 juillet 1586 (indications figurant respectivement dans le Prologue et à la dernière page, $M A: 5,483$ ), pendant les années que son auteur passa dans l'Audiencia de Quito et dans celle de Lima. L'oeuvre ne fut pas publiée du vivant de Cabello mais plusieurs indices montrent bien qu'elle circula sous forme manuscrite (7). L'histoire de ses éditions — bien que normale pour des textes de l'époque, surtout dans les vice-royautés - en dit beaucoup sur l'accueil reçu et sur la façon dont l'oeuvre a été jugée et étudiée, c'est-à-dire en tant que mine de renseignements sur l'histoire de l'empire incasique et sa conquête (8).

(4) Il est urgent d'en faire une nouvelle édition. Il nous a été impossible de consulter les ms. connus (l'autographe de la Bibliothèque de l'Université du Texas à Austin ou la copie de la New-York Public Library). Nos affirmations dépendent donc de l'édition de 1951 que nous citons tout au long de l'article en utilisant l'abréviation $M A$.

(5) Primera parte/ dela Misce/lanea Austral/ de don Diego D'Avalos y/ Figueroa, en varios co-/loquios. Interlocutores; Delio, y Cilena./Con la Defensa de Damas./Dirigida al escellentissimo/ señor Don Luys de Velasco, Cauallero dela Orden de Santiago./Visorey, y Capitan general de los Reynos del Piru./Chile, y Tierra firme./Con licencia de sv Excelencia/Impresso en Lima por Antonio Ricardo. Año/ M.DC.II.

(6) Francisco Pa-/tricio de Reyno, y de/ la institvcion del que / Ha de Reynar, y de como deue auerse con los subdi-/tos, y ellos con el. Donde se traen notables exemplos,/ è historias, y dichos agudos, y peregrinos. Mate-/ria gustosissima para todo genero de gentes./Traduzido por Henrique Garces de/ Latin en Castellano./ Dirigido a Philippo/Segvndo deste nombre, y primer Monarca/ de las Españas, y de las Indias./[armoiries]/ Con privilegio./ En Madrid, por Luis Sanchez./ M.D.XCI. L'oeuvre de Patrizzi est un traité pour l'éducation du Prince, et si nous la mentionnons dans ce contexte, c'est parce qu'elle partage avec un grand nombre d'ouvrages de l'époque (et comme le révèle le soustitre) le fait d'être à caractère miscellanique.

(7) Qu'elle ait été citée par la Poetisa Anónima dans une oeuvre publiée en 1608 (voir note 13), par Antonio de León Pinelo dans son Epítome de la Biblioteca Oriental y Occidental de 1629 et par Antonio de la Calancha dans sa Corónica moralizada del orden de San Agustín en el Perú de 1639, démontre que l'oeuvre circula sous forme manuscrite.

(8) La première édition de la Miscelánea, partielle, fut une traduction en français, éditée par Henri de Ternaux-Compans, de la troisième partie, à partir du chapitre IX, là où commence l'histoire 
Le jugement négatif de l'ouvrage de la part de la critique contemporaine en raison de la variété de la matière présentée n'est pas anormal (9) mais plutôt commun àl' accueil réservé aux miscellanées, y compris la primitive Silva de varia lección (1540) de Pedro Mexía, il y a encore relativement peu de temps. Malgré l'énorme succès d'édition que l'oeuvre connut à l'époque, c'est le jugement si souvent répété de Marcel Bataillon contre l'auteur sévillan — jugement qui ne fut pas isolé, exprimant une opinion courante de la part de la critique — qui a prévalu dans l'appréciation moderne de l'oeuvre (10). Les études qui l'envisagent en tenant compte des paramètres de l'époque et du genre, et qui ont conduit à une juste appréciation de sa valeur et de son influence sont relativement récentes. En effet, les miscellanées semblent éloignées des préoccupations du critique actuel ; en outre, il s'agit de textes dont l'approche n'est pas exempte de difficultés, causes principales de l'abandon dans lequel ils ont été laissés (11). C'est particulièrement vrai pour le domaine hispano-américain où très souvent font défaut des études abordant le texte en fonction du milieu culturel et idéologique de sa création (et non pas en fonction de nos préoccupations politiques). Des oeuvres comme les miscellanées sont cependant fondamentales pour comprendre la culture de l'Humanisme et les idées qui circulaient parmi les érudits, comme Cabello Valboa, et les artistes de l'époque. Homme à l'esprit curieux et très intéressé par les vieilles histoires de la terre où il passa la plus grande partie de sa vie, Cabello semble avoir réalisé, tout en menant une vie des plus errantes, une production littéraire importante (12). Loué par l'auteur du "Discurso en loor de la poesía", dont la valeur programmatique dans le projet de l'Académie Antarctique est connu (13), il semblerait qu'il se soit exercé dans plusieurs genres : nous sont parvenus ses miscellanées et ses relaciones (1945b); ont été perdus

de l'empire des Incas (1840, vol. XV). La première édition espagnole (partielle) fut une retraduction en espagnol de la précédente (1920, tome II). L'oeuvre ne fut intégralement éditée qu'en 1945 par Jacinto Jijón y Caamaño (1945a). Édition basée sur le ms. de la New-York Public Library.

(9) Porras juge anodin et manquant d'originalité tout ce qui ne concerne pas l'histoire indigène, faisant allusion à l' "énorme poids mort d'érudition que représentent les chapitres sur l'origine de l'homme américain et les longues et indigestes énumérations de faits historiques européens sur les papes, les rois médiévaux ou les empereurs byzantins" (Porras Barrenechea, 1986 : 682-683).

(10)“... livre médiocre [...] le type même du pot-pourri qui faisait les délices des robustes appétits de cette époque". "Le lecteur, comme l'auteur, y nage dans l'océan d'une curiosité sans préférences, sans idées, $s$ 'accrochant successivement aux dépouilles les plus hétérogènes." (Bataillon, 1982[1950] : 637-638).

(11) Se reporter aux propos pertinents de Isaías Lerner dans un article consacré au sousgenre : $1998: 71-82$. Au sujet du manque d'intérêt actuel pour les miscellanées, voir p. 79.

(12) La plus grande partie des renseignements que nous avons sur sa vie proviennent de son oeuvre. On sait qu'il naquit à Archidona (Málaga) entre 1530 et 1535 , qu'il alla se battre dans les Flandres dans les armées espagnoles et qu'il arriva aux Indes Occidentales en 1566. De la Nouvelle Grenade il passa dans l'Audiencia de Quito où il fut ordonné prêtre (1571) et où il demeura, occupant diverses doctrinas. En 1580 nous le retrouvons dans l'Audiencia de Lima, dans sa paroisse de San Juan Bautista à Ica, bien qu'il se rende souvent à Lima et dans le Nord. Finalement, nous savons qu'il s'installa dans l'Audiencia de Charcas. Voir Tauro del Pino, 1948 : 172-178.

(13) Le "Discurso" fut publié dans les préliminaires de la Primera Parte del Parnaso antártico de obras amatorias de Diego Mexía de Fernangil (Sevilla, 1608); pour Cabello, voir fol. 20 v. Pour la valeur programmatique du "Discurso", voir Colombí-Monguió (2000). 
la "Volcánea", le "militar elogio", la "Comedia del Cuzco y Vasquirana” (14). Au sein de ce projet de translatio studii qui fut celui de l'Académie Antarctique, la figure de Cabello est de premier plan et sa miscellanée un texte ambitieux, d'abord pour avoir choisi un genre humaniste qui, en tant que recueil du savoir, suppose le maniement d'un abondant matériel érudit, bien qu'il ne soit pas de première main, et ensuite parce qu'il traite un sujet polémique et central concernant les Indes Occidentales. Laissant donc de côté la qualité de l'oeuvre en tant que source historique, notre but est de restituer l'oeuvre dans le cadre des idées (alimenté par la varietas) et dans le genre choisi (la miscellanée), en la mettant en rapport avec une famille de textes avec lesquels elle dialogue et en la plaçant dans une tradition qui lui donne un sens.

L'auteur conçoit son oeuvre comme une miscellanée et, comme nous le verrons plus loin, il l'inscrit expressément dans ce genre (15). Qu'est-ce que cela impliquait d'écrire une miscellanée - et le faire depuis les Indes Occidentales — au XVIe siècle ? La miscellanée a été définie comme "l'oeuvre ou l'écrit où sont traitées un grand nombre de matières confuses et mélangées" (Diccionario de Autoridades). Il s'agit d'un genre qui n'avait pas été abordé par la théorie poétique classique et de la Renaissance. Les auteurs qui s'y consacraient suivaient, jusqu'à un certain point, les modèles classiques existants qui pouvaient être rédigés en prose ou sous forme de dialogue, parmi lesquels on en distingue quatre : l'Histoire naturelle de Pline, les Nuits attiques d'Aulu-Gelle, les Saturnales de Macrobe et le Banquet des sophistes d'Athénée (16). N'étant pas soumis à un mode d'énonciation, à un programme, à une longueur ou à une structure particulière, l'auteur de miscellanée disposa d'une très grande liberté de composition ; il s'ensuit que les oeuvres que nous considérons comme faisant partie du genre varient énormément, au point que le mélange d'un matériel divers et souvent sans rapport entre ses éléments semble être le seul dénominateur commun(17). Néanmoins, en prenant les modèles classiques mentionnés et les oeuvres de la Renaissance que nous

(14) Leur existence nous est connue par la mention qu'en fait la Poetisa Anónima dans le "Discurso" (voir note 13). On peut supposer, d'après leurs titres, que les deux premières oeuvres étaient des poèmes épiques ou, dans le cas de la seconde, un panégyrique, et que la dernière (ou les dernières car on n'est pas sûr qu'il ne s'agisse pas de deux ouvrages différents) était une comedia.

(15) Cabello explique la signification géographique qu'il donne à "antarctique", comme synonyme d'austral, c'est à dire appartenant aux régions du Sud et par opposition à "boréal” $(M A: 4)$. Pour une analyse détaillée de la question, voir Firbas (2000).

(16) Voir Lerner. L'inclusion de l'oeuvre de Pline, bien que ce ne soit pas une miscellanée, se justifie par sa valeur en tant que modèle et mine de renseignements pour les auteurs de la Renaissance (1998: 72).

(17) On peut le constater en passant en revue le contenu des modèles classiques : les Saturnales de Macrobe englobe la religion antique, la mythologie, l' histoire de Rome, la physique, la physiologie, la conduite humaine ; le Banquet des sophistes d'Athénée, la philosophie, la caractérologie, la musique, l'histoire, la médecine, l'histoire naturelle ; les Nuits attiques d'Aulu-Gelle portent sur le droit, la grammaire, les antiquités, l'histoire et la biographie, la critique textuelle et littéraire, relatant en outre les excursions à des lieux fameux et des repas avec ses pupilles. Pour la miscellanée de la Renaissance, voir la note suivante. 
pouvons qualifier de miscellanées (18), il est possible de déterminer une série d'aspects communs. La diversité des thèmes et l'accumulation encyclopédique semblent être les caractéristiques fondamentales, auxquelles on peut ajouter les suivantes : conception des chapitres en unités fermées et indépendantes mais ordonnées de telle façon qu'il existe une certaine concaténation ; alternance de thèmes graves ou superficiels; brièveté dans le traitement de la question ; préférence pour l'insolite, l'inconnu, tout ce qui est ingénieux ou pouvant servir d'exemple (19).

Dans la Miscelánea Antártica de Cabello certains de ces aspects sont absents car elle n'est pas structurée en chapitres courts, autonomes et interchangeables dont le thème embrasse une portion variée du savoir. Au contraire, et comme nous le verrons plus loin, il s'agit d'une oeuvre ayant un thème central fort et polémique, développé en suivant un fil conducteur qui va du général et éloigné dans le temps et l'espace, au particulier et contemporain à l'auteur et aux lecteurs. C'est peut-être pour cette raison qu'elle n'a pas été lue comme une miscellanée mais comme une histoire des Incas avec un énorme et gênant pourcentage de matière bâtarde. Cependant, la matière considérée comme bâtarde et le déploiement d'érudition répondent à la structure que l'auteur imprima à son oeuvre, laquelle répond à une vision plurielle de l'univers et à une conception du savoir alimentée par le concept de varietas.

Sur le plan rhétorique la variété remplit une fonction précise. La miscellanée est un genre utilisé par les humanistes pour sa capacité à toucher un grand public auquel on peut ainsi transmettre un savoir. Pour que ce but soit atteint le lecteur doit avant tout se sentir attiré par la matière qui lui est présentée, raison pour laquelle on cherche à éveiller sa curiosité, à l'étonner et à le surprendre avec des faits notables et des histoires singulières (Rallo Gruss, 1984 : 160). C'est donc par la variété de la matière que l'auteur tente de capter l'intérêt du lecteur, sans pour cela se voir obligé d'enfreindre le voeu de véracité qu'il a formulé (bien que parfois il préfère le faire).

Dès le premier paragraphe de son prologue, Cabello annonce à son lecteur qu'il lui fournira un matériel vrai, de l'Histoire, mais qu'en même temps ce sera plaisant du fait de la variété qu'elle comporte :

(18) Lerner (1998 : 75-77) considère comme miscellanées les oeuvres suivantes : Polidoro Vergilioi Urbinato, De Rerum inventoribus libri octo (1502), qui se limite à l'origine de toutes les choses imaginables; Raffaele Maffei (Volaterrano), Commentariorum Rerum Urbanorum Libri XXX (Roma, 1506), qui traite en trois parties de Géographie, Moeurs et Philologie ; Battista Fulgoso, De dictisfactisque memorabilibus Collectanea (1509), sur des thèmes variés incluant des chapitres sur les cultes religieux, les institutions anciennes, les traits de caractère, etc. ; Joannes Ravisius Textor, Officina (2a éd. 1532, dictionnaire de citations classiques comprenant 350 sections classées par thème ; Ludovico Ricchieri (Caelius) qui tente de reconstituer dans son Antiquarum lectionum commentarios (1516) une miscellanée perdue et commence selon un certain ordre (création du monde, les dieux, les hommes, etc.), lequel est rompu à partir du livre III ; Alessandro ab Alessandro, Dies geniales (1522). Ceci dit, il est clair que la frontière entre miscellanée, polyanthée et autres répertoires n'est guère précise. Sur leur prolifération, voir les articles de Infantes $(1988 ; 2000)$.

(19) Antonio Castro, dans sa riche introduction à la Silva de Pedro Mexía, a signalé les caractéristiques précédentes mais en se basant précisément sur cette oeuvre (1989: 65 et suiv.). Rallo Gruss avait défini la miscellanée comme une "somme de thèmes sans ordre rigoureux, à la manière de chapitres indépendants" (1984: 170). 
“Il n'y a rien de mieux adapté au goût des Lecteurs (comme l'écrit Cicéron à son ami Lucceius) que l'histoire qui en soi contient des variétés dans les temps et des changements de fortune dans les événements ; car pour nos esprits il est plaisant et amusant de lire de telles choses (bien que nous ne souhaitions pas en faire l'expérience) et la remémoration sûre du travail passé apporte de la douceur et de la délectation.” (MA : 3) (20).

Il est intéressant qu'il ait choisi cette citation et pas une autre, celle où Cicéron met l'accent non pas sur le caractère didactique de l'histoire mais sur le pouvoir qu'elle a de capter l'esprit du lecteur, sur sa capacité à "recréer les entendements", précisément par les fluctuations que subissent les événements et les personnages dans l'espace et dans le temps : le texte de Cabello, emprunté à une épître de Cicéron à Lucceius, suit presque textuellement l'original. Dans cette épître, Cicéron lui suggère de séparer son action politique de l'Histoire des guerres civiles qu'il était en train de rédiger et d'en faire un volume à part. Comme argument pour le convaincre il lui dit que les événements qu'il a vécus portent en eux une infinie variété, ce qui lui permettra de capter et de maintenir l'attention du lecteur car, ajoute-t-il, il n'y a rien de mieux pour faire plaisir au lecteur que les multiples changements de circonstance et les viscissitudes de la fortune. Bien que personne ne les désire pour soi-même, quand on les lit ils sont source d'intérêt pour ceux qui les ont vécus car se remémorer les adversités passées ne manque pas de charme ; pour ceux qui ne les ont pas subis il existe le plaisir d'être spectateur des souffrances des autres et de pouvoir sentir de la compassion pour eux (21). Exciter l'esprit du lecteur, l'incliner vers l'admiration ou le mépris, faire qu'il suive les fortunes et adversités des personnages (d'Adam à Atahualpa, en passant par Sémiramis et Curicuillor) en sympathisant avec eux ou en fustigeant leurs actes, l'auteur peut y parvenir grâce à la variation intrinsèque du devenir historique. Cependant, le matériel historique remplit également une autre fonction primordiale : celle d'instruire dans la matière des Indes Occidentales, en ce qui concerne la nature (en présentant des cas surprenants et des curiosités) et les activités de l'être humain (en traitant l'un des thèmes polémiques de son époque, l'origine de l'homme américain). Ainsi, la variété qui nourrit l'oeuvre ne surgit pas de l'ampleur de la thématique traitée, comme ce fut le cas dans plusieurs des modèles classiques et dans la Silva de Mexía (22), mais du thème principal, comme le fait apparaître le titre :

(20) "No ay cosa mas adaptada á el gusto de los Lectores (dice Ciceron escriviendo a su amigo Luceyo) que la historia que en si contiene variedades en los tiempos, y mutaciones de fortuna en los subcesos; porque las tales cosas (aunque no deseemos experimentarlas) es grato, y jocundo à nuestros entendimiento el leerlas : que la recordacion segura, de el passado trabajo, trae consigo dulcedumbre, y delectacion." (MA:3).

(21) Epistulae ad familiares, V, XII, 4-5 : "Multiam etiam casus nostri tibi varietatem in scibendo suppeditabunt, plenam cuiusdam voluptatis, quae vehementer animos hominum in legendo te scriptore tenere possit. Nihil est enim aptius ad delectationem lectoris, quam temporum varietates fortunae que vicissitudines; quae etsi nobis optabiles in experiendo non fuerunt, in legendo tamen erunt iucundae. Habet enim praeteriti doloris secura recordatio delectationem. Ceteris vero, nulla perfunctis propria molestia, casus autem alienos sine ullo dolore intuentibus, etiam ipsa misericordia est iucunda.", in : Ciceron, 1965[1927].

(22) De Courcelles a proposé de manière convaincante l'existence d'un principe articulateur de type idéologique pour l'oeuvre de Mexía in : de Courcelles (2000). 
Miscelannée antarctique, où est décrite l'origine de nos Indiens Occidentaux depuis Adam et la fondation et commencement de l'empire des rois Ingas du Pérou, vies et guerres qu'ils eurent : choses notables qu'ils firent, comptées les années de leurs naissances et de leurs morts, et ce qui survenait dans l'univers ; pendant leurs années de vie et leurs époques" (23).

L'existence du thème central se répète dans le prologue où l'auteur annonce qu'il va relater "l'origine et le commencement dans le Monde de nos Indiens Occidentaux" $(M A: 3)$. Dans la suite, pourtant, il n'attache plus d'importance à cette apparente unité thématique de l'oeuvre en montrant à son lecteur — s'appuyant sur une autre référence à Ciceron qui va dans le même sens que la précédente — que le développement d'un tel thème exige, du fait de son étendue dans l'espace et le temps, de "forcément [...] passer par de nombreuses variations de temps, et d'altérations de fortune" (MA:3). C'est en raison de cette variété, émanant de la matière même, que l'oeuvre s'intitule miscellanée :

“ce n'est pas sans l'avoir bien pensé qu'a été donné à notre histoire le nom de Miscellanée, à cause de cette mixture forcée d'histoires que la ligne de son procédé implique : que le faire est obligé (comme tu le verras) et qu'en plus le mélange de tant de couleurs embellira son discours" $(M A: 3)$.

C'est donc cette "mixture forcée" que Cabello annonce à son lecteur comme attrait de son oeuvre, lui promettant une variété encore plus grande grâce à l'ajout d'anecdotes et à l'ornement que comporte son discours :

"ces Histoires ne sont pas si sèches ni dénuées de fleurs et de couleurs très plaisantes au point de ne plus produire douceur et délectation à l'esprit humain..." (MA:8) (24).

Le caractère miscellanique de l'oeuvre est en outre mis en relief dans l'en-tête de chacune des trois parties — s'ajoutant à la sommaire description du contenu — qui incluent "d'autres curiosités concernant chaque province et dignes d'être connues" (1e partie) ; "d'autres curiosités singulières dignes d'êtres connues des curieux" (2e partie); "beaucoup de choses surprenantes qu'il y a au Pérou" (3e partie). Comme c'est le cas dans les autres miscellanées, l'accent est mis sur la nouveauté et l'insolite de l'information proposée, de même que, comme l'indique clairement le titre de la troisième partie, sur l'étonnement qu'on espère provoquer chez le lecteur, étonnement qui, nous l'avons vu, est considéré par les humanistes comme étant le premier pas sur le chemin du savoir.

(23) "MISCELANEA/ Antartica, donde se describe, el origen, de nuestros Indios Occide[n]/ tales, deduzido desde Adan, y la Erection y principio del Imperio de los Reyes Ingas de el Piru Vidas y guerras que tu/vieron : cossas notables q[u]e hizieron, computados los años de sus/ nascimientos y muertes, y de lo q[u]e por el Uniuersso yva subcedi/endo; durantes [sic] sus edades y tiempos". Tiré du fac-similé de la page de titre du ms. d'Austin, reproduite dans l'édition de 1951.

(24) "no sin maduro acuerdo le fue puesto a nuestra historia el nombre de Miscelanea, por la forzoza mixtura, de historias que consigo antecoge, el hilo de su proceder: que demas de ser lance forzozo el hacerlo (como bien veras) hermoseara mucho su discurso la mixtura de tanto colores" $(M A: 3 \mathrm{~s})$. 
Enfin, la variété de la matière et son caractère encyclopédique, de même que l'unité mentionnée dans la ligne d'argumentation, se voit clairement quand on passe en revue le contenu de la Miscelánea Antártica:

La première partie (13 chap., 70 p.) commence par la création du monde, donne une chronologie des temps anciens et relate l'épisode de Caïn, pour passer ensuite à Noé, se concentrant à partir de là et durant plusieurs chapitres sur la répartition du monde après la séparation des langues dans l'épisode biblique de la Tour de Babel ; sont abordées ensuite l'origine des Noirs, l'évangélisation que la reine de Saba et le PrêtreJean auraient réalisée en Afrique et, finalement, Ofir est proposé comme "père des Indiens" (chap. 12).

La seconde partie (20 chap., 120 p.) débute par l'invention des lettres par Adam et se poursuit par la démonstration selon laquelle les Indiens descendraient du Patriarche Ofir (chap. 3), réfutant la possibilité d'une origine israélite ou carthagénoise (chap. 4 et 5), tout en signalant que les Israélites connaissaient l'existence des Indes Occidentales et que Salomon avait envoyé des flottes vers le continent (chap. 3 et 7); on relate ensuite comment les Indiens arrivèrent aux Indes (chap. 15), comment ils se multiplièrent (chap. 18), quelle est l'origine des Indiens de la Nouvelle-Espagne (chap. 18), de ceux du Chili et des Chiriguanos (chap. 19), pour conclure, après avoir comparé leurs rites, coutumes et cérémonies, que ces derniers descendent des Indiens orientaux. Tout cela parsemé de chapitres où sont rapportées "les grandeurs de l'Inde Orientale, avec les tyrannies les plus notables qui survinrent alors", ou bien traitant de la reine Sémiramis, de la naissance de Moïse, des guerres de Cyrus, de la naissance et des campagnes d'Alexandre, etc.

Quant à la troisième partie (33 chap. 273 p.), les premiers chapitres traitent de cas surprenants : si les monstres auxquels les Anciens se réfèrent existent (chap. 2) ; pourquoi il ne pleut pas sur la côte péruvienne et "autres curiosités et secrets de la nature" (chap. 4); les volcans (chap. 5). On y parle aussi de la navigation ancienne et moderne (chap. 1); de la venue au monde du Christ et de ce qu'il advint des idoles (chap. 3) ; de la répartition des terres entre les Indiens (chap. 4); de la barbarie dans laquelle vivaient les Indiens ; de la façon dont ils se remémorent leurs histoires (chap. 6) ; des Apôtres qui reçurent la mission d'aller prêcher de par le monde ; de l'action du Démon, d'Arrien et d'autres hérésies (chap. 7) ; des empereurs catholiques, de Mahomet et de savoir comment l'islam aurait pénétré dans les Indes Occidentales si les Rois Catholiques ne l'avaient pas empêché (chap. 8). Bien que l'arrivée des Indiens sur les terres péruviennes ait été racontée (chap. 4 et 6), ce n'est qu'au chapitre 9 que commence le fil narratif qui se prolongera jusqu'à la fin, l'histoire des Incas, quoique toujours relatée en contrepoint des événements universels contemporains et des faits notables : après la question de l'état de barbarie dans lequel vivaient les Indiens avant l'arrivée des Incas (chap. 9) viennent les gouvernements des divers Incas (jusqu'au chap. 22), la guerre entre Huáscar et Atahualpa et l'arrivée des Espagnols (chap. 23 à 33).

Comme on a pu le constater dans le résumé précédent, Cabello s'efforce d'introduire la variété, y compris dans la troisième partie, celle qui sans aucun doute présente la plus grande unité(25). Primo, et suivant le schéma que propose de façon plus

(25) À une unité spatiale (la plupart des événements rapportés surviennent au Pérou), s'ajoute un lien chronologique et souvent causal à partir de la narration du gouvernement des Incas (chap. 9) et une unité thématique (le triomphe de la foi chrétienne sur l'idolâtrie, l'islam et l'hérésie). 
ou moins ordonnée un grand nombre de chroniques des Indes, il nous situe du point de vue de l'espace et du climat sur le territoire que les Incas auraient à dominer, mais toujours en s'arrêtant sur la chose insolite ou ignorée qu' on y rencontre (26). Secundo, la matière bâtarde ne manque pas, bien au contraire (27). Tertio, c'est ici qu'est relatée, bien que brièvement, l'histoire d'Efquen Pisan et de Chestan Xectuin et, longuement, celle de Quilaco et Curicuillor, matière amoureuse que l'auteur inclut, selon sa déclaration expresse, pour apporter la variété que requiert une miscellanée :

“On parle tant de ce Jeune Homme bien né, à cause de l'étrange aventure qui lui advint en chemin durant ce voyage, aventure qui est digne de faire partie de notre histoire parce que - afin que justement cette oeuvre puisse bénéficier du nom de Miscellanée - il est nécessaire que toutes les matières y soient traitées et qu'il nous manque la matière amoureuse, laquelle jusqu'à cette circonstance ne s'était pas présentée et nous arrive maintenant en abondance et doit être introduite dans la trame de notre toile, en apportant quelque douceur parmi tant d'aigreur et d'amertume comme dans ce qui nous reste à écrire." (MA : Chap. 4) (28)

Enfin, la variation est à la base de la méthode de son enquête qui consiste à opposer, chaque fois que c'est possible, les événements historiques survenant plus ou moins en même temps dans le monde. C'est ce qu'il a expliqué dans le prologue où il promet dans la seconde partie (perdue ou jamais rédigée), centrée en grande partie sur l'Asie, de ne pas oublier non plus "les choses dignes de mémoire qui survenaient dans notre Espagne pendant les années et les temps que nous considérerons" ( $M A: 8)$.

Le même procédé est maintenu dans la troisième partie, comme il l'indique dans le prologue, en utilisant la métaphore de l'oeuvre semblable à une toile que l'auteur tisse petit à petit :

"et cette plaisante toile tu la trouveras nuancée et retouchée avec les événements les plus remarquables qui survenaient en même temps dans les trois principales parties qui constituent notre monde" (MA:8).

En effet, l'histoire des Incas est mise en parallèle avec les événements universels qui lui sont contemporains, en particulier ceux qui ont lieu en Europe (chap. 10-14, 19, $20,23,24)$, ce qui oblige Cabello à couper le fil de sa narration et à se transporter dans

(26) Comme exemple du caractère de miscellanée de cette troisième partie, cela vaut la peine de donner le titre de deux des chapitres : "Où l'on discute et l'on conclut par des raisons naturelles, s'il y a eu, s'il y a ou s'il peut y avoir dans le monde les monstres, ainsi que l'écrivent les Auteurs. C'est un chapitre digne d'être remarqué par les curieux" (MA : chap. 2) ; "des causes pour lesquelles il ne pleut pas dans les plaines du Pérou et des conditions et complexions des Indiens et autres curiosités et secrets de la nature" (MA : chap. 4).

(27) À celle qu'apporte, dans les premiers chapitres, la description déjà mentionnée des phénomènes naturels, s'ajoute par exemple, dans le chap. 11, l'origine des gitans, etc.

(28) "Acese tanta relacion deste bien nacido Joven, por el estraño caso que en este viage y camino le subcedio, el qual se porna en historia por ser digno della y tambien por que para que justamente esta obra pueda gozar del nombre de Miscelanea es necesario que en ella se traten todas materias, y faltanos la amorosa que hasta esta coyuntura no se nos a ofrecido, y hasta ahora que tan a manos llenas nos llega a quento interponerse ha en la tela de nuestro estambre siruiendo de alguna dulzura entre tanto agro y amargo como nos resta en lo que queda por escriuir". (MA, chap. 26 : 408). 
l'espace (29). La troisième partie narrée (à partir du chap. 9) sur le modèle humaniste de la biographie des gouvernants, dont les vies s'ordonnent chronologiquement et dont les événements, inévitablement, s'enchaînent souvent de façon causale présente une plus grande unité aux yeux du lecteur. Cette unité se voit renforcée à partir du moment où Cabello commence à relater ce qui lui est presque contemporain (ou au moins contemporain aux sources qu'il utilise), c'est-à-dire les guerres entre Huáscar et Atahualpa. C'est à partir de là (chap. 26) qu'il abandonne la méthode jusqu'alors maintenue du parallèle historique, s'en tenant à un seul fil de l'histoire ; par ailleurs, c'est précisément dans ce chapitre qu'il commence àrelater l'histoire de Quilaco et Curicuillor, comme on l'a vu, dans le but exprès de ne pas négliger la constante variété dans la matière qu'exige la miscellanée (Rose, 1999 ; 2000).

Sa méthode de travail en parallèle lui a permis d'atteindre un autre objectif de la miscellanée, objectif qui répond à la vision universaliste de l'humanisme chrétien : compléter un domaine connu avec tout ce qui est éloigné, aussi bien dans le temps que dans l'espace. Dans cette perspective, et à travers la mosaïque de savoir universel que construit le genre miscellanique, l'homme "s'intègre dans le monde, à partir de sa connaissance globale et spécifique, et pas seulement dans son environnement quotidien" (Rallo Gruss, 1984 : 160, 162). Dans ce sens, la Miscelánea Antártica de Cabello fonctionne sur deux plans différents. Il est certain qu'elle fait entrer le lecteur, tant local que péninsulaire, dans un milieu qui lui est totalement ou partiellement inconnu, celui de la matière américaine, mais en même temps elle assimile celle-ci au fonds commun du savoir universel. La variété naturelle et humaine nouvellement découverte dans les territoires d'outre-mer constitue pour Cabello, comme ce le fut pour Torquemada, la preuve de l'existence de Dieu et de son plan divin : l'histoire indigène étant rattachée sur un plan d'égalité à l'histoire universelle, l'Indien devient un être historique. Doté d'origines et d'avenir, apparenté à ses congénères par des liens formés par Cabello, il est intégré dans la carte humaine : créé par Dieu, marqué par le péché originel, relié à son Créateur par le pacte de Noé et, grâce à la conquête, ouvert au salut, sa différence accidentelle se fond dans l'égalité de son essence humaine devant Dieu.

(29) Voir par exemple le chap. 10 : "De ce que l'on tient pour vrai à propos du début et de l'origine des rois Ingas et des choses qui survenaient dans le monde connu quand ces Princes commencèrent à régner et de la descendance des Turcs et autres curiosités". Juan Gil avait déjà signalé l'intention de Cabello d'insérer l'histoire des Incas dans l'Histoire universelle, bien qu'(Gil) ne dévellopa cette idée(1989 : 300). 


\section{Références citées}

BATAILLON, M., 1982[1950] - Erasmo y España. Estudios sobre la historia espiritual del siglo $X V I$; México : Fondo de Cultura Económica.

CABELlo VALBOA, M., 1840 - Histoire du Pérou par Miguel Cavello Balboa. Inédite. In : Voyages, relations et mémoires, vol. XV (Henri de Ternaux-Compans, édt) ; Paris : Arthur Bertrand.

CABELLO VALBOA, M., 1920 - Historia del Perú bajo la dominación de los Incas por el R.P. Miguel Cabello Balboa (escrita de 1576 a 1586). Anotaciones y concordancias con las crónicas de Indias de Horacio Urteaga. Biografía de Cabello Balboa de Carlos A. Romero. In : Colección de libros y documentos referentes a la Historia del Perú ; Lima : Imprenta y Librería Sanmarti, 2e série, tome II. Traduction de Delia Rosa Romero.

CABELlO VALBOA, M., 1945a - Miscelánea antártica. In : Obras (Jacinto Jijón y Caamaño, édit.) : 91-443 ; Quito : Editorial Ecuatoriana.

CABELLO VALBOA, M., 1945b - Verdadera descripción y relación de la provincia y tierra de las Esmeraldas. Orden y traza para descubrir y poblar la tierra de los chunchos y otras provincias. In : Obras (Jacinto Jijón y Caamaño, édit.) ; Quito : Editorial Ecuatoriana.

CABELlo VAlBOA, M., 1951 - Miscelánea antártica. Una historia del Perú antiguo ; Lima : Universidad Nacional Mayor de San Marcos.

CALANCHA de la, A., 1639 - Corónica moralizada del Orden de San Agustín en el Perú ; Barcelona : P. Lacavallería.

CASTRO, A., 1989 - Introducción a Pedro Mexía, Silva de varia lección ; Madrid : Cátedra, 2 vols.

CICERON, M. T., 1965[1927) - Epistulae ad familiares. In : The letters to his friends. With an English translation by W. Glynn Williams; :M.A.London-Cambridge(Mass.) :HeinemannHarvard University Press.

COLOMBÍ-MONGUIÓ de, A., 2000. Erudición humanista en saber omnicomprensivo e identidad colonial. In : La formación de la cultura virreinal. I : La etapa inicial : 75-91. Études réunies par Karl Kohut et Sonia V. Rose ; Madrid : Iberoamericana.

DÁVALOS Y FIGUEROA de, D., 1602-1603 - Primera parte de la Miscelanea Austral ; Lima : Antonio Ricardo.

COURCELLES de, D., 2000 - La Silva de varia lección, de l'humaniste sévillan Pedro Mexía ou l'échec du principe de varietas. In : Fonder les savoirs, fonder les pouvoirs, XVe-XVIIe siècles : 81-95 ; Paris : École des chartes. Travaux réunis et présentés par Dominique de Courcelles.

COURCELLES de, D., 2001 - Varietas/De varietate rerum à la Renaissance ; Paris : École des chartes. Études réunies par Dominique de Courcelles

FIRBAS, P., 2000 - Escribir en los confines : épica colonial y mundo antártico. In : Agencias criollas. La ambigüedad colonial en las letras hispanoamericanas : 191-213 ; Pittsburg : Instituto Internacional de Literatura Iberoamericana. Études réunies par José Antonio Mazzoti.

GIL, J., 1989 - Mitos y utopías del descubrimiento. Vol. 3 : El Dorado ; Madrid : Alianza.

INFANTES, V., 1988 - De officinas y polyantheas: los diccionarios secretos del Siglo de Oro. In : Homenaje a Eugenio Asensio : 243-257 ; Madrid : Gredos.

LEÓN PINELO de, A., 1629 - Epítome de la Biblioteca Oriental y Occidental ; Madrid : Iuan Gonzalez.

LEÓN PINELO de, A., 1737-1730 - Epítome de la Biblioteca Oriental y Occidental ; Madrid. Édition augmentée par Andrés González de Barcia.

LERNER, I., 1998 - Misceláneas y polianteas del Siglo de Oro español. In : Actas del Congreso Internacional sobre Humanismo y Renacimiento (Juan Matas Caballero et al.) : 71-82 ; León : Universidad de León. 
LÓPEZ POZA, S., 1990 - Florilegios, polianteas, repertorios de sentencias y lugares comunes. Aproximación bibliográfica. Criticón, 49: 61-76.

LÓPEZ POZA, S., 2000 - Polianteas y otros repertorios de utilidad para la edición de textos del Siglo de Oro. La Perinola, 4 : 191-214.

MEXÍA DE FERNANGIL de, D., 1608 - Primera Parte del Parnaso antártico de obras amatorias; Sevilla : Gamarra.

MEXÍA, P., 1989 - Silva de varia lecicón; Madrid : Cátedra, 2 vols.

PATRIZZI, F., 1591 - De Reyno, y de la institvcion del que ha de Reynar... Traduzido por Henrique Garces de Latin en Castellano ; Madrid : Luis Sánchez.

Poetisa anonima. Discurso en loor de la Poesía. Voir Mexía de Fernangil.

PORRAS BARRENECHEA, R., 1986 - La Miscelánea Antártica de Cabello Balboa. (Compte rendu de l'édition de Jijón y Caamaño, 1945). In : Los cronistas del Perú y otros ensayos : 682-683; Lima: Banco de Crédito. Édition, prologue et notes de Franklin Pease G.Y.

RALLO GRUSS, A., 1984 - Las misceláneas, conformación y desarrollo de un género renacentista. Edad de Oro, III : 159-180.

RODRÍGUEZCACHO, L., 1991 - Del silencio y la curiosidad sobre América en las Misceláneas. Edad de Oro, $\mathbf{X}: 167-186$.

ROSE, S. V., 1999 - Los amores de Quilaco Yupanqui y la hermosa Curicuillor: raigambre europea de una historia de tema incaico. In : Transgressions et stratégies du métissage en Amérique coloniale (travaux réunis et présentés par Bernard Lavallé) : 119-138; Paris : Presses Universitaires de la Sorbonne Nouvelle.

ROSE, S. V., 2000 - Una historia de linajes a la morisca: los amores de Quilaco y Curicuillor en la Miscelánea Antártica de Cabello Valboa. In : La formación de la cultura iberoamericana virreinal. I : La etapa inicial (travaux réunis par K. Kohut et S.V. Rose) : 189-212 ; Madrid : Iberoamericana.

TAURO DEL PINO, A., 1948 - Esquividad y gloria de la Academia Antártica ; Lima: Huascarán. TORQUEMADA de, A., 1982 - Jardín de flores curiosas... ; Madrid : Castalia. Édition de Giovanni Allegra. 